\title{
Experimental and Theoretical Studies of Hydroxyethyl Cellulose as Inhibitor for Acid Corrosion Inhibition of Mild Steel and Aluminium
}

\author{
I.O. Arukalam ${ }^{*}, 1$ I.C. Madufor ${ }^{1}$, O. Ogbobe ${ }^{1}$ and E. Oguzie ${ }^{2}$ \\ ${ }^{I}$ Department of Polymer and Textile Engineering, Federal University of Technology, P.M.B. 1526, Owerri, Nigeria \\ ${ }^{2}$ Electrochemistry and Materials Science Research Laboratory, Department of Chemistry, Federal University of \\ Technology, P.M.B. 1526, Owerri, Nigeria
}

\begin{abstract}
Hydroxyethyl cellulose (HEC) was studied as corrosion inhibitor for mild steel and aluminium in $0.5 \mathrm{M} \mathrm{H}_{2} \mathrm{SO}_{4}$ solution using weight loss and electrochemical techniques. The results revealed that HEC inhibited corrosion of mild steel and aluminium in sulphuric acid environment. Inhibition efficiency was found to depend on concentration of the inhibitor. Impedance results revealed that HEC was adsorbed on the corroding metal surface while polarization results show that HEC inhibited both anodic and cathodic reactions of mild steel but showed anodic dissolution reaction on aluminium due to destabilization of the passive oxide film on the Al surface and inhibited only cathodic reaction. Inhibition of corrosion reaction in the acidic medium by adsorption on the metal/solution interface follows Langmuir and Freundlich adsorption isotherms. Also, inhibition efficiency increased with rise in temperature. Local reactivity of the HEC molecule was analyzed theoretically using the density functional theory. The theoretical predictions showed good agreement with experimental results.
\end{abstract}

Keywords: Acid inhibition, EIS, aluminium, mild steel, polarization, weight loss.

\section{INTRODUCTION}

Metals and alloys are exposed to the action of acids in industrial processes where acids play important roles such as in oil well acidizing, acid pickling, acid cleaning and acid descaling [1]. The effects of the acids on metals could be destructive leading to metal corrosion. Corrosion inhibitors are usually added to the acid solutions to minimize metal loss and reduce acid consumption [2-7]. However, some of these corrosion inhibitors are very inimical to public health [8-12] and this has necessitated the search for eco-friendly corrosion inhibitors for metals in acid solutions.

Many researchers have studied the effect of some synthetic organic inhibitors on the corrosion of steel in sulphuric and hydrochloric acid solutions with positive results [13-20]. The exploitation of polymers as corrosion inhibitors has recently attracted considerable attention. The corrosion inhibition of low carbon steel in phosphoric acid by polyvinylpyrrolidone (PVP) and polyethyleneimine (PEI) as inhibitors has been reported [21]. Polarization and weight loss studies showed that both polyvinylpyrrolidone and polyethyleneimine are effective for the inhibition of low carbon steel over a wide range of aqueous phosphoric acid solutions. Corrosion inhibition of mild steel in sulphuric acid solution using polyethylene glycol methyl ether (PEGME) has been reported using electrochemical polarization (galvanostatic and potentiostatic) techniques [22]. It was

*Address correspondence to this author at the Department of Polymer and Textile Engineering, Federal University of Technology, P.M.B. 1526, Owerri, Nigeria; Tel: +234 703042 0870;

E-mail: innocentarukalam@yahoo.com found that PEGME is a very effective corrosion inhibitor for mild steel in acidic medium. The inhibiting power of these polymers is related structurally to the aromatic rings, presence of conjugation and hetero-atoms (oxygen, sulphur, phosphorus and nitrogen) that are the major functional centres of adsorption [23].

Hydroxyethyl cellulose is widely used in cosmetics, as well as cleaning solutions and other household products. Presently, to the best of our knowledge, there is no reported work on inhibitive effects of hydroxyethyl cellulose on corrosion of mild steel and aluminium in sulphuric acid solution. Therefore, we consider it necessary to study the effect of hydroxyethyl cellulose on acid corrosion inhibition of mild steel and aluminium in sulphuric acid.

\section{MATERIALS AND METHODS}

Hydroxyethyl cellulose sourced from Sigma-Aldrich, Fluka analytical, Germany was used as obtained, and four different concentrations of the inhibitor solutions ranging from $(500 \mathrm{mg} / \mathrm{L}-2000 \mathrm{mg} / \mathrm{L})$ was prepared. Sulphuric acid used was of BDH AR grade. 0.5 $\mathrm{M} \mathrm{H}_{2} \mathrm{SO}_{4}$ solution was also prepared and used as corrodent. Mild steel coupons having weight-percentage composition of $\mathrm{C}=0.04, \mathrm{Si}=0.02, \mathrm{Mn}=$ $0.04, \mathrm{Cu}=0.06, \mathrm{Cr}=0.05$, and remainder $\mathrm{Fe}$ and aluminium sheets of the type AA 1060 and purity $98 \%$ were used in this study. The coupons were used as cut without further polishing but were however degreased in absolute ethanol, dried in acetone and weighed. Weight loss measurements were conducted on rectangular mild steel and aluminium coupons of dimension $2 \mathrm{~cm} \times 4 \mathrm{~cm} \times 0.2 \mathrm{~cm}$, immersed in 200 $\mathrm{ml}$ of test solutions. The coupons were retrieved at $24-\mathrm{h}$ interval progressively for 5 days. At the end of each 
immersion time interval, the coupons were retrieved and immersed in $20 \% \mathrm{NaOH}$ solution containing $200 \mathrm{~g} / \mathrm{L}$ of zinc dust, scrubbed with bristle brush, washed, dried and reweighed. This was done to stop further corrosion reaction. The weight loss was calculated as the difference between the final weight at a given time and the initial weight. The values recorded were mean values of triplicate determinations. Thereafter, the values of corrosion rate were calculated from the following equation [24]:

$C R(\mathrm{~mm} / \mathrm{yr})=\frac{87,600 \mathrm{~W}}{\rho A t}$

where $W$ is the weight loss in gram (g), $\rho$ the density of the mild steel coupon $\left(\mathrm{g} / \mathrm{cm}^{3}\right), A$ the exposed surface area of the coupon $\left(\mathrm{cm}^{2}\right)$ and $t$ the exposed time (h).

The percentage inhibition efficiency (I.E.\%) was calculated using the equations:

$$
\text { I.E. } \%=\left(1-\frac{C R_{\text {inh }}}{C R_{\text {blank }}}\right) x 100
$$

where $C R_{\text {inh }}$ and $C R_{\text {blank }}$ are corrosion rates in the presence and absence of inhibitor respectively.

Electrochemical tests were carried out in a conventional three-electrode configuration with graphite rod as counter electrode and saturated calomel electrode (SCE) as the reference electrode. The working electrode $\left(1 \mathrm{~cm}^{2}\right.$ surface area) was immersed in the test solution and after establishing a steady state OCP (open circuit potential), the electrochemical measurements were carried out in a computer controlled electrochemical workstation (VERSASTAT3 Model). Electrochemical impedance spectroscopy (EIS) measurements were carried out in frequency range of $10 \mathrm{KHz}$ to $10 \mathrm{~Hz}$ with amplitude of $10 \mathrm{mV}$ using a.c. signals at open circuit potential. The potentiodynamic polarization curves are obtained in the potential range $-250 \mathrm{mV}$ to $+250 \mathrm{mV}$ for mild steel and $250 \mathrm{mV}$ to $+1600 \mathrm{mV}$ for aluminium with a sweep rate of 0.5 $\mathrm{mV} / \mathrm{s}^{-1}$.

Quantum chemical computations were performed using the density functional theory (DFT) electronic structure programs -Forcite and $\mathrm{DMol}^{3}$ as contained in Materials Studio 4.0 software (Accelrys Inc.). The electronic structure of HEC on Fe and Al surfaces was modeled by means of the
DFT electronic structure program $\mathrm{DMol}^{3}$. Electronic parameters for the simulation include restricted spin polarization using DNP basis set and Perdew Wang (PW) local correlation density functional. The distribution of frontier molecular orbitals, namely, the highest occupied molecular orbital (HOMO) and lowest unoccupied molecular orbital (LUMO) and chemical reactivity were evaluated by taking into account the Fukui indices. Fe $\left(\begin{array}{lll}1 & 1 & 0\end{array}\right)$ was chosen to simulate the adsorption process of HEC on mild steel and Al $\left(\begin{array}{lll}1 & 1 & 0\end{array}\right)$ for aluminium. The molecular dynamics simulation was performed at $298 \mathrm{~K}$ using NVE ensemble, with a time step of $0.1 \mathrm{fs}$ and simulation time of $50 \mathrm{ps}$.

\section{RESULTS AND DISCUSSION}

\subsection{Weight Loss Measurements}

A number of mechanistic studies on the anodic dissolution of iron in acidic sulphate solutions have been undertaken, and the hydroxyl accelerated mechanism proposed initially by Bockris and Drazic [25] and reported by Oguzie [26] has gained wide acceptance:

$$
\begin{aligned}
& \mathrm{Fe}+\mathrm{OH}^{-} \hat{\rightarrow} \mathrm{FeOH}_{a d s}+\mathrm{H}^{+}+e^{-} \\
& \mathrm{FeOH}_{a d s} \stackrel{r d s}{\longrightarrow} \mathrm{FeOH}^{+}+e^{-} \\
& \mathrm{FeOH}^{+}+\mathrm{H}^{+} \rightrightarrows \mathrm{Fe}^{2+}+\mathrm{H}_{2} \mathrm{O}
\end{aligned}
$$

As a consequence of these reactions, including the high solubility of the corrosion products, the metal loses weight in the solution. Corrosion inhibition is initiated by the displacement of adsorbed water molecules by inhibitor species leading to the specific adsorption of the inhibitor on the metal surface [2].

The calculated values of corrosion rates and inhibition efficiencies for mild steel and aluminium dissolution in 0.5 $\mathrm{M} \mathrm{H}_{2} \mathrm{SO}_{4}$ from weight loss measurements are presented in Tables 1 and 2 respectively. The inhibition efficiency of $\mathrm{HEC}$ on mild steel corrosion in $0.5 \mathrm{M} \mathrm{H}_{2} \mathrm{SO}_{4}$ is illustrated in Fig. (1). The plots show that inhibition efficiency increased with increasing concentration of HEC. The maximum inhibition efficiency is $93.99 \%$ at $2000 \mathrm{mg} / \mathrm{L} \mathrm{HEC}$.

Naturally, aluminium has self-protective oxide film. When in aggressive solution such as sulphuric acid, the stability of the passive film is interfered, rendering it less protective and allowing the molecules of the corrodent to diffuse to the active sites on the aluminium surface.

Table 1. Calculated Values of Corrosion Rates (mm/yr) and Inhibition Efficiency (I.E.\%) for Mild Steel Corrosion in $\mathrm{O.5} \mathrm{M}_{2} \mathrm{H}_{2} \mathrm{SO}_{4}$ from Weight Loss Measurements

\begin{tabular}{|c|c|c|c|c|c|c|c|c|c|c|}
\hline & \multicolumn{4}{|c|}{$\begin{array}{c}\text { Corrosion Rate (mm/yr) } \\
\text { Day }\end{array}$} & \multicolumn{4}{c|}{ Inhibition Efficiency (I.E.\%) } \\
Day \\
\hline \hline Conc. (mg/L) & 1 & 2 & 3 & 4 & 5 & 1 & 2 & 3 & 4 & 5 \\
\hline Blank & 2.39 & 2.51 & 2.18 & 1.91 & 1.73 & - & - & - & - & - \\
\hline $\mathbf{5 0 0}$ & 2.31 & 1.49 & 1.18 & 0.99 & 0.90 & 9.06 & 43.95 & 48.75 & 50.93 & 51.20 \\
\hline $\mathbf{1 0 0 0}$ & 0.88 & 0.86 & 0.72 & 0.60 & 0.61 & 62.89 & 65.57 & 66.52 & 68.45 & 64.37 \\
\hline $\mathbf{1 5 0 0}$ & 0.78 & 0.55 & 0.39 & 0.34 & 0.32 & 67.85 & 78.18 & 82.15 & 82.24 & 93.61 \\
\hline $\mathbf{2 0 0 0}$ & 0.49 & 0.19 & 0.14 & 0.12 & 0.12 & 80.58 & 92.69 & 93.99 & 93.90 & 93.61 \\
\hline
\end{tabular}


Inhibition or retardation of corrosion is achieved by incorporating compounds that have the capability to deactivate the corrodents and reducing their rate of diffusion to the metal surface.

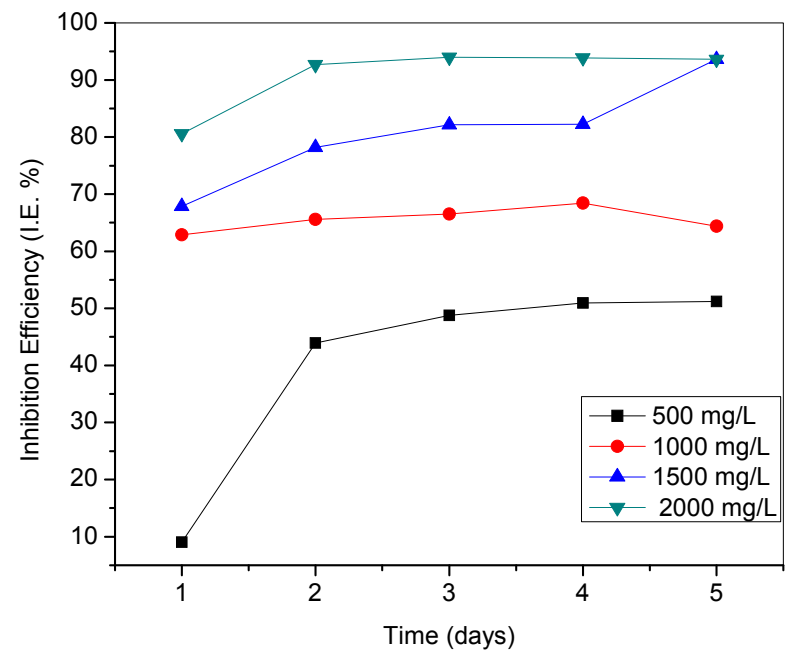

Fig. (1). Inhibition efficiency against time for mild steel corrosion in $0.5 \mathrm{M} \mathrm{H}_{2} \mathrm{SO}_{4}$, in the absence and presence of different concentrations of HEC.

Fig. (2) clearly shows that the inhibitive effect of HEC on aluminium corrosion in $0.5 \mathrm{M} \mathrm{H}_{2} \mathrm{SO}_{4}$ solution is also concentration dependent. The maximum inhibition efficiency of $\mathrm{HEC}$ for aluminium corrosion in $0.5 \mathrm{M} \mathrm{H}_{2} \mathrm{SO}_{4}$ is $65.52 \%$ at the highest concentration of HEC investigated. These results indicate that inhibitive capability of HEC is more efficient on mild steel than on aluminium.

Sequel to the mechanistic approach reported above by Oguzie, Ashassi-Sorkhabi and Nabavi-Amri [27] proposed the following mechanism involving two adsorbed intermediates to account for the retardation of $\mathrm{Fe}$ anodic dissolution in the presence of an inhibitor:

$$
\begin{aligned}
& \mathrm{Fe}+\mathrm{H}_{2} \mathrm{O} \rightarrow \mathrm{Fe} . \mathrm{H}_{2} \mathrm{O}_{a d s} \\
& \mathrm{Fe} . \mathrm{H}_{2} \mathrm{O}_{a d s}+\mathrm{Y} \rightarrow \mathrm{FeOH}_{a d s}^{-}+\mathrm{H}^{+}+\mathrm{Y} \\
& \mathrm{Fe} . \mathrm{H}_{2} \mathrm{O}_{a d s}+\mathrm{Y} \rightarrow \mathrm{FeY}_{a d s}+\mathrm{H}_{2} \mathrm{O} \\
& \mathrm{FeOH}{ }_{\text {ads }}^{-} \stackrel{r d s}{\longrightarrow} \mathrm{FeOH}_{\text {ads }}+e^{-}
\end{aligned}
$$

$$
\begin{aligned}
& \mathrm{FeY}_{a d s} \leftrightarrows \mathrm{FeY}^{+}+e^{-} \\
& \mathrm{FeOH}_{\text {ads }}+\mathrm{FeY}_{a d s}^{+} \rightrightarrows \mathrm{FeY}_{a d s}+\mathrm{FeOH}^{+} \\
& \mathrm{FeOH}^{+}+\mathrm{H}^{+} \rightrightarrows \mathrm{Fe}^{2+}+\mathrm{H}_{2} \mathrm{O}
\end{aligned}
$$

where Y represents the inhibitor system.

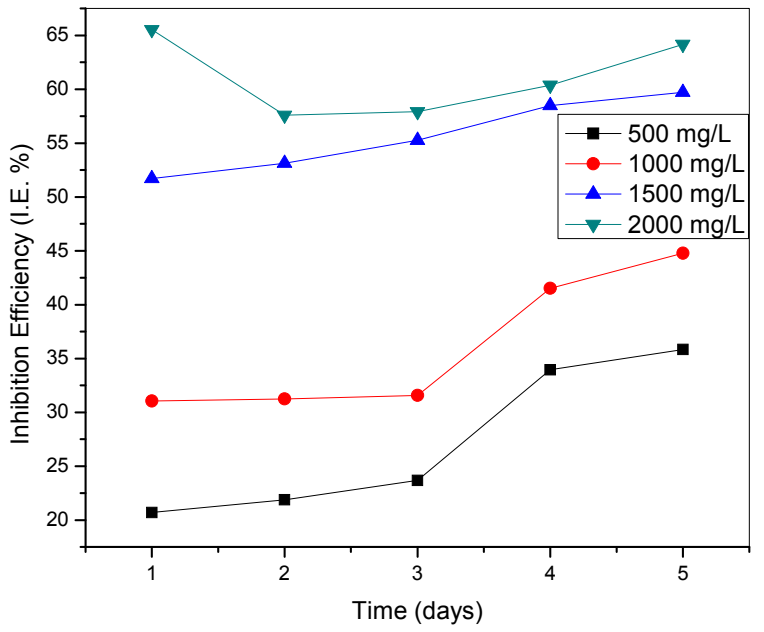

Fig. (2). Inhibition efficiency against time (days) for aluminium corrosion in $0.5 \mathrm{M} \mathrm{H}_{2} \mathrm{SO}_{4}$, in the absence and presence of different concentrations of HEC.

If we consider the non-homogeneous nature of metal surfaces arising from the existence of lattice defects and dislocations, a corroding metal surface is generally characterized by multiple adsorption centres having activation energies and heats of adsorption. Inhibitor molecules may thus be adsorbed more readily at surface active sites having suitable adsorption enthalpies. Considering the detailed mechanism above, displacement of some adsorbed water molecules on the metal surface by inhibitor species to yield the adsorbed intermediate $\mathrm{FeY}_{\text {ads }}$ (Eqn.4c) lowers the amount of the species $\mathrm{FeOH}_{\text {ads }}^{-}$available for the rate-determining steps and resultantly suppresses $\mathrm{Fe}$ anodic dissolution.

Further inspection of the plots (Figs. 1, 2) shows the variation of inhibition efficiency against immersion time. It is found that IE\% increases gradually with time for both mild steel and aluminium dissolution in $0.5 \mathrm{M} \mathrm{H}_{2} \mathrm{SO}_{4}$ solution.

Table 2. Calculated Values of Corrosion Rates ( $\mathrm{mm} / \mathrm{yr}$ ) and Inhibition Efficiency ( $I . E . \%$ ) for Aluminium Corrosion in $0.5 \mathrm{M}$ $\mathrm{H}_{2} \mathrm{SO}_{4}$ from Weight Loss Measurements

\begin{tabular}{|c|c|c|c|c|c|c|c|c|c|c|}
\hline & \multicolumn{4}{|c|}{$\begin{array}{c}\text { Corrosion Rate (mm/yr) } \\
\text { Day }\end{array}$} & \multicolumn{5}{c|}{$\begin{array}{c}\text { Inhibition Efficiency (I.E.\%) } \\
\text { Day }\end{array}$} \\
\hline \hline Conc. (mg/L) & 1 & 2 & 3 & 4 & 5 & 1 & 2 & 3 & 4 & 5 \\
\hline Blank & 0.21 & 0.12 & 0.09 & 0.10 & 0.09 & - & - & - & - & - \\
\hline $\mathbf{5 0 0}$ & 0.17 & 0.09 & 0.07 & 0.07 & 0.06 & 20.69 & 21.88 & 23.68 & 33.96 & 35.82 \\
\hline $\mathbf{1 0 0 0}$ & 0.15 & 0.08 & 0.07 & 0.06 & 0.06 & 31.03 & 31.25 & 31.58 & 41.51 & 44.78 \\
\hline $\mathbf{1 5 0 0}$ & 0.10 & 0.05 & 0.04 & 0.04 & 0.04 & 51.72 & 53.13 & 55.26 & 58.49 & 59.70 \\
\hline $\mathbf{2 0 0 0}$ & 0.08 & 0.05 & 0.04 & 0.04 & 0.04 & 65.52 & 57.58 & 57.90 & 60.38 & 64.18 \\
\hline
\end{tabular}


Table 3. Impedance and Polarization Parameters for (a) Mild Steel and (b) Aluminium Corrosion in $0.5 \mathrm{M} \mathrm{H}_{2} \mathrm{SO}_{4}$

\begin{tabular}{|c|c|c|c|c|c|c|}
\hline HEC (mg/L) & $R_{c t}\left(\Omega c m^{2}\right)$ & $C_{d l}\left(\mu \boldsymbol{F c m}^{-2}\right) \times 10^{-3}$ & $n$ & $E_{\text {corr }}(\mathbf{m V}(\mathbf{S C E}))$ & $I_{\text {corr }}\left(\mu \mathrm{Acm}^{-2}\right)$ & $I E_{R c t} \%$ \\
\hline (a)Blank & 9.434 & 16.87 & 0.8949 & -463.54 & 1711.5 & - \\
\hline HEC & 49.55 & 3.21 & 0.8631 & -478.72 & 396.46 & 73.06 \\
\hline $\mathrm{HEC}+\mathrm{KI}$ & 129.8 & 1.23 & 0.8154 & -479.10 & 124.2 & 92.73 \\
\hline (b)Blank & 289.4 & 0.55 & 0.9137 & -1154.4 & 4.9715 & - \\
\hline HEC & 1829 & 0.09 & 0.9132 & -650.30 & 47.417 & 84.18 \\
\hline $\mathrm{HEC}+\mathrm{KI}$ & 388.1 & 0.41 & 0.9289 & -671.31 & 45.299 & 25.43 \\
\hline
\end{tabular}

However, Fig. (2) shows that for $2000 \mathrm{mg} / \mathrm{L} \mathrm{HEC}$, the trend possibly suggests higher IE\% at $\mathrm{t}$ (time) less than $24-\mathrm{h}$. This result corroborated the EIS findings. This behaviour is consequent upon the fact that polymer dissolution is a gradual process, and it takes time for the HEC molecules to be fully solvated. As the solvation increases with time, the dispersion of the HEC molecules enabled their adsorption onto the metal surfaces. Hence, there is geometric blocking or deactivation of the active sites, leading to increase in inhibition efficiency.

\subsection{Electrochemical Impedance Spectroscopy Results}

The effect of HEC on the corrosion behaviour of mild steel and aluminium in $0.5 \mathrm{M} \mathrm{H}_{2} \mathrm{SO}_{4}$ was studied by EIS measurements at room temperature after an exposure period of 30 minutes (time take to reach open circuit potential) and results are presented in Table $\mathbf{3}$.

Figs. $(3,4)$ show the Nyquist plots of the impedance data as depressed capacitive semicircles. The diameter found by the intercept of each of these loops and the horizontal $Z_{\text {real }}$ axis is related to the charge transfer resistance $\left(R_{c t}\right)$. The value of charge transfer resistance obtained is a measure of electron transfer across the metal/solution interface and is inversely proportional to corrosion rate. Hence, a large $R_{c t}$ value indicates strong resistance against corrosion. $R_{c t}$ values were obtained after modeling the impedance data using an appropriate equivalent circuit [R(Q)R] contained in ZSimpWin3.21 software. The inhibition efficiency was calculated from the relation shown in equation (5):

I.E.\% $=\left(1-\frac{R_{c t}}{R_{c t i n h}}\right) x 100$

where $R_{c t}$ and $R_{\dot{a} n h}$ are the charge transfer resistance without and with addition of inhibitor respectively.

$R_{c t}$ of $9.434 \Omega \mathrm{cm}^{-2}$ was obtained for the uninhibited solution. In the presence of HEC inhibitor, higher value of $R_{c t}, 49.55 \Omega \mathrm{cm}^{-2}$ was observed indicating that $\mathrm{HEC}$ molecule inhibited mild steel corrosion in $0.5 \mathrm{M} \mathrm{H}_{2} \mathrm{SO}_{4}$. With halide salt addition, it was clearly noticed that the impedance spectrum was of largest capacitive loop, having $R_{c t}$ value of $129.8 \Omega \mathrm{cm}^{-2}$ indicating very high inhibition efficiency.

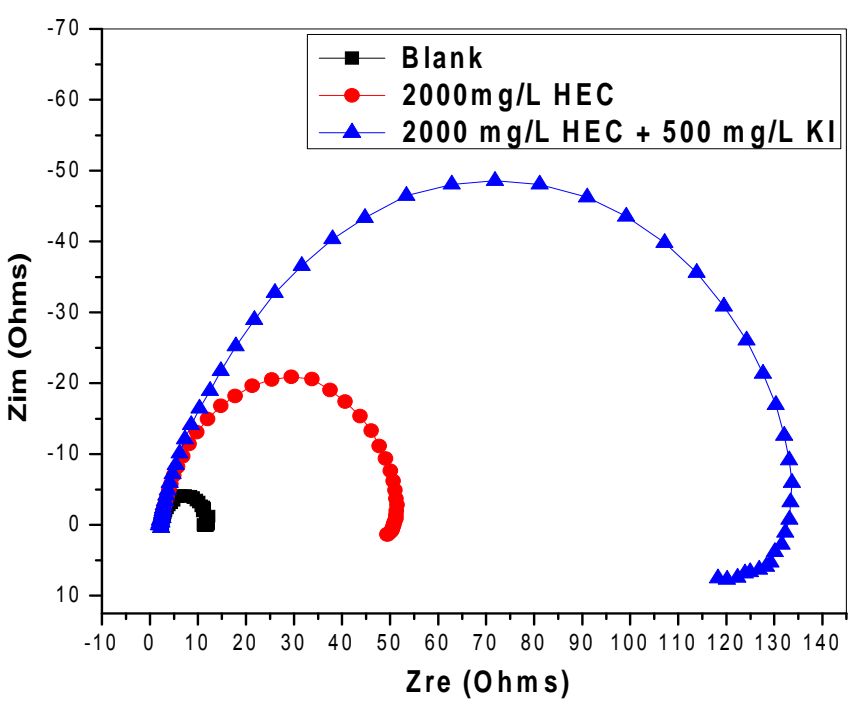

Fig. (3). Nyquist Impedance plot of mild steel corrosion in $0.5 \mathrm{M}$ $\mathrm{H}_{2} \mathrm{SO}_{4}$ for HEC, in the absence and presence of KI.

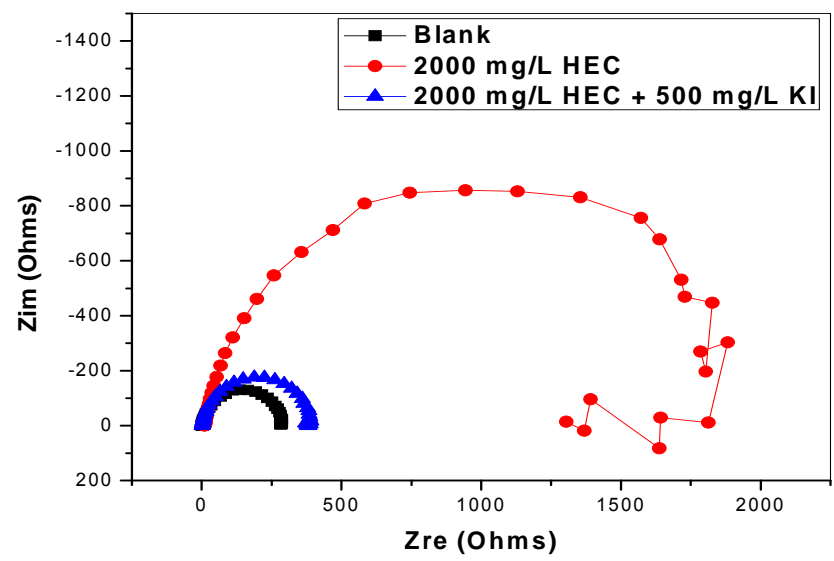

Fig. (4). Nyquist Impedance plot of Aluminium corrosion in $0.5 \mathrm{M}$ $\mathrm{H}_{2} \mathrm{SO}_{4}$ for HEC, in the absence and presence of KI.

Fig. (4) shows that HEC inhibited aluminium corrosion. The increase in charge transfer resistance in the presence of HEC arise from the change in chemical composition of the surface film due to incorporation of HEC inhibitor molecules 
into the film especially through defect sites with low ionic resistance. The incorporated molecules form high resistive compounds [28-30]. Since the anodic reaction rate is determined by the diffusion of ions through the surface film it can be reasoned that the formation of resistive compounds in the film gave rise to the increase in the charge transfer resistance which is responsible for inhibition. However, in the presence of $\mathrm{HEC}+\mathrm{KI}$, the $\mathrm{R}_{\mathrm{ct}}$ value decreased. Correspondingly, the thickness of the interfacial layer decreased, leading to reduction of the inhibition efficiency. This result explains that KI acted antagonistically with HEC.

Inhibition efficiencies of $73.06 \%$ and $92.73 \%$ were obtained on mild steel by HEC and HEC+ KI respectively, while HEC gave $84.18 \%$ IE and HEC+KI, $25.43 \%$ for aluminium dissolution in sulphuric acid solution.

These results suggest inhibition performance of HEC on corrosion of mild steel and aluminium in the acid solution. This observation shows that the corrosion of mild steel and aluminium in acid solution is mainly controlled by a charge transfer process [19]. The deviation from ideal semicircle is generally attributed to the frequency dispersion as well as to the surface irregularity of the metals. This disparity could be explained by non-ideal behaviour of double layer as a capacitor. Therefore, it is necessary to use a constant phase element, CPE instead of double layer capacitor to account for non-ideal behaviour. The CPE is defined in impedance form as [36]:

$Z_{C P E}=A^{-1}(j \omega)^{-n}$

where $A$ is the $C P E$ constant, $\omega$ is the angular frequency $(\mathrm{rad} / \mathrm{s}), j^{2}=-1$ is the imaginary number and $n$ is a $C P E$ exponent which can be used as a measure of the heterogeneity or roughness of the surface. The double layer capacitance values were calculated using the expression:

$C_{d l}=\frac{1}{2 \pi f_{\max } R_{c t}}$

The impedance data for the Nyquist plots were appropriately analyzed by fitting to the equivalent circuit $R_{s}\left(C_{d l} R_{c t}\right)$, (Fig. 5) which has previously been used to model Fe/acid or $\mathrm{Al} /$ acid interface $[26,28,36]$.

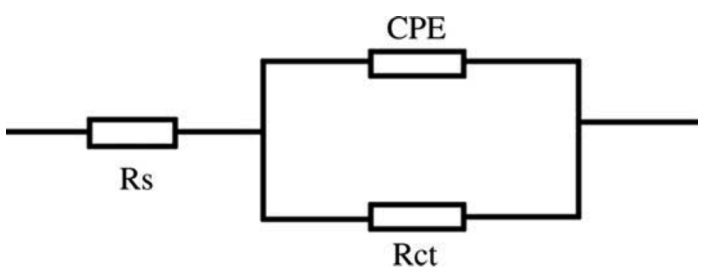

Fig. (5). Equivalent circuit used to fit the mild steel or aluminium impedance spectra.

The corresponding electrochemical parameters given in Table 3 reveal that incorporation of HEC into the acid corrodents caused the charge transfer resistance to increase, while reducing the double layer capacitance. This effect becomes more pronounced on addition of KI to HEC, for mild steel corrosion whereas the addition of KI to HEC showed antagonistic effect for aluminium dissolution. The increase in the $R_{c t}$ values points toward enhanced corrosion resistance due to the corrosion-inhibiting potential of HEC. The decrease in $C_{d l}$ values, resulting from a decrease in the dielectric constant and/or increase in the interfacial thickness, can be attributed to the adsorption of HEC onto the metal/solution interface and reduces the corrosion rate of the metals in the acid corrodent. This assertion can be justified by Helmholtz model [36] which is given as

$C_{d l}=\varepsilon \varepsilon_{o} A / \delta$

where $\varepsilon$ and $\varepsilon_{o}$ are the dielectric constant of the medium and permittivity of vacuum respectively, while $A$ is the surface area of the electrode and $\delta$ the thickness of the adsorbed interfacial layer.

\subsection{Potentiodynamic Polarization Results}

The calculated values of polarization parameters are presented in Table 3. Fig. (6) presents the anodic and cathodic polarization curves for mild steel corrosion in $0.5 \mathrm{M}$ $\mathrm{H}_{2} \mathrm{SO}_{4}$ in the absence and presence of HEC and HEC $+\mathrm{KI}$. The plots indicate that the anodic and cathodic reactions in the blank acid follow Tafel's law. The inhibited solution containing $2000 \mathrm{mg} / \mathrm{L}$ HEC produced a pronounced effect shifting the $\mathrm{E}_{\mathrm{corr}}$ in the anodic direction and a very high inhibiting effect was observed on the anodic reaction sites. For inhibited solution containing $2000 \mathrm{mg} / \mathrm{L}$ HEC +500 $\mathrm{mg} / \mathrm{L} \mathrm{KI}, \mathrm{E}_{\text {corr }}$ was not notably affected while the cathodic and anodic branches were shifted to lower values of corrosion current, though the former effect was more pronounced. These results indicate that HEC+KI is a mixedtype inhibitor with predominant cathodic effect.

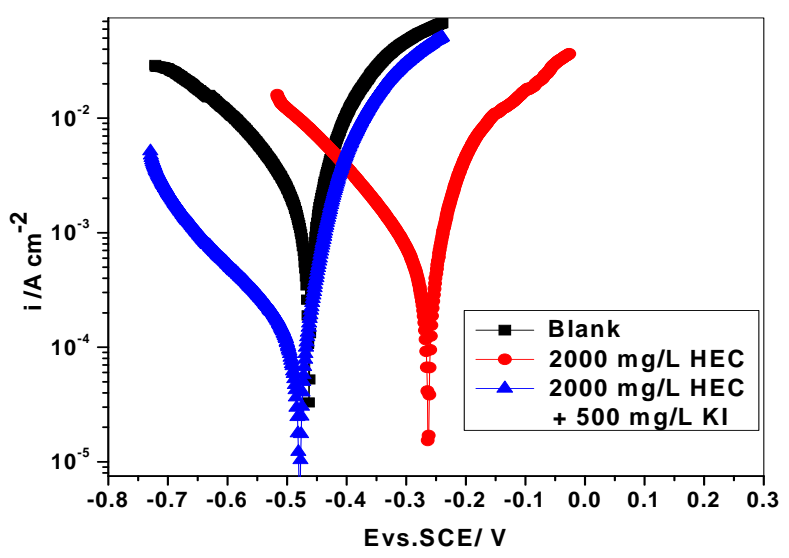

Fig. (6). Polarization curves for mild steel corrosion in $0.5 \mathrm{M}$ $\mathrm{H}_{2} \mathrm{SO}_{4}$ using HEC, in the absence and presence of KI.

Fig. (7) illustrates the anodic and cathodic polarization curves of aluminium dissolution in $0.5 \mathrm{M} \mathrm{H}_{2} \mathrm{SO}_{4}$ in the presence of HEC and HEC+KI. The polarization behaviour of Aluminium in the presence of HEC and HEC + KI shows two distinct conflicting effects. In the anodic region, the inhibitor systems obviously interfered with the stability of the passive film, rendering it less protective, with the resultant increase of the anodic current density. This effect is very much pronounced in the presence of KI due to the well known adverse influence of halogens on passivity. The 
inhibitor systems however exert an inhibiting effect in the cathodic domain, thereby reducing the cathodic current density. This cathodic inhibiting effect is thus responsible for the overall inhibiting effect of the HEC and HEC+KI as observed from weight loss and impedance measurements. From point of view of corrosion inhibition performance, it could be reasoned that the presence of the passive oxide film interferes with (or actually restricts) HEC adsorption under the given conditions, thereby limiting the inhibiting effect to more cathodic potentials where the absence of oxide film would allow the formation of a polymeric film of HEC.

\subsection{Adsorption Consideration}

The adsorption of an organic inhibitor on the surface of the corroding metals observed from the impedance data may be regarded as a substitution process between the inhibitor and water molecules adsorbed on the metal surface. Addition of inhibitor molecules adsorbs on the metal surface and interaction between them can be described by adsorption isotherms [31]. HEC adsorption on mild steel surface was found to follow the adsorption isotherm of Freundlich, given by equation (9):

$\theta=K C^{n}$

The plot of $\log \theta$ versus $\log C$ gives a straight line (Fig. 8) indicating that the adsorption of HEC on mild steel surface from $0.5 \mathrm{M} \mathrm{H}_{2} \mathrm{SO}_{4}$ follow Freundlich isotherm. Adherence to Freundlich isotherm is further evidence of the adsorption of HEC on the mild steel surface.

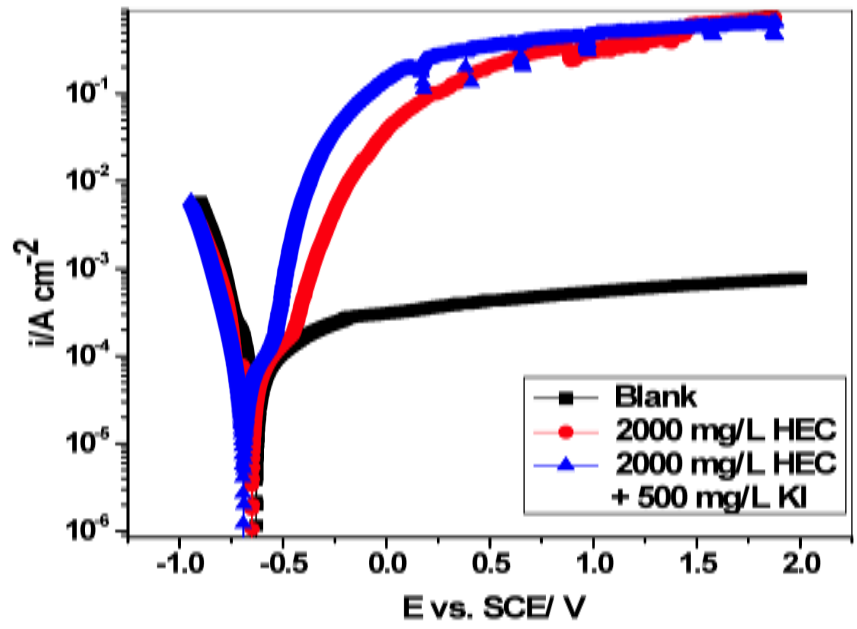

Fig. (7). Polarization curves of Aluminium corrosion in $0.5 \mathrm{M}$ $\mathrm{H}_{2} \mathrm{SO}_{4}$ using HEC, in the absence and presence of KI.

The adsorption of HEC on aluminium was found to follow Langmuir isotherm which is based on the assumption that all adsorption sites are equivalent and that particle binding occurs independently from nearby sites being occupied or not [32]. A correlation between surface coverage $(\theta)$ and the concentration $(C)$ of inhibitor in the electrolyte can be represented by the Langmuir adsorption isotherm:

$C / \theta=1 / K+C$

where $K$ is equilibrium adsorption constant. The linear plot obtained in Fig. (9) indicates that the adsorbing HEC species occupies typical adsorption site at the metal/solution interface [33].

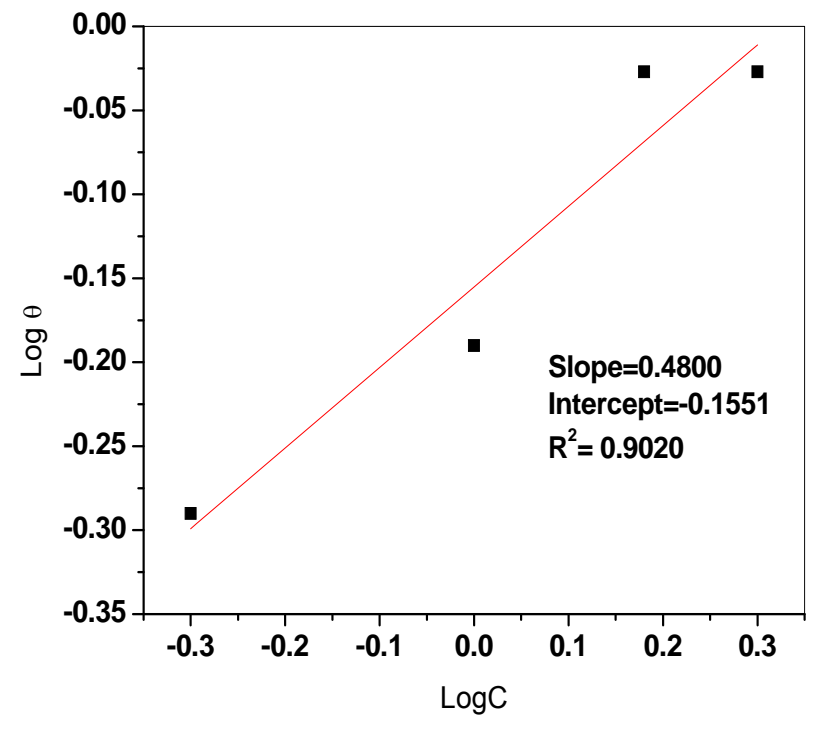

Fig. (8). Freundlich isotherms for HEC adsorption on mild steel in $0.5 \mathrm{M} \mathrm{H}_{2} \mathrm{SO}_{4}$ solution.

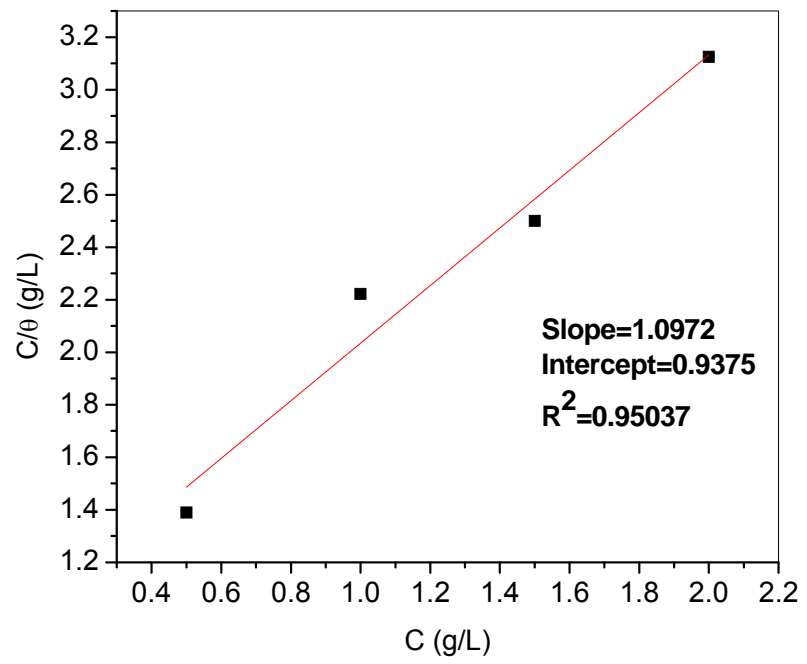

Fig. (9). Langmuir isotherm for HEC adsorption on Aluminium in $0.5 \mathrm{M} \mathrm{H}_{2} \mathrm{SO}_{4}$ solution.

In general, $K$, the equilibrium adsorption constant, represents the adsorption power or binding strength of the inhibitor molecule on the metal surface. Results from Figs. $(8,9)$ show that $K$ values are 1.07 and 0.70 for aluminium and mild steel respectively. The positive values confirm the adsorbability of HEC on the metal surfaces.

\subsection{Effect of Temperature}

To evaluate the effect of temperature on corrosion and corrosion inhibition process, weight loss measurements were performed at $10 \mathrm{~K}$ intervals in the temperature range $303-$ $333 \mathrm{~K}$ in uninhibited acid $\left(0.5 \mathrm{M} \mathrm{H}_{2} \mathrm{SO}_{4}\right)$ and in inhibited solutions containing $500 \mathrm{mg} / \mathrm{L}$ and $2000 \mathrm{mg} / \mathrm{L} \mathrm{HEC} \mathrm{for} \mathrm{mild}$ steel and aluminium dissolution in $0.5 \mathrm{M} \mathrm{H}_{2} \mathrm{SO}_{4}$. The results obtained are shown in Figs. $(\mathbf{1 0}, \mathbf{1 1})$ for mild steel and aluminium respectively. Increase in temperature, increased the inhibition efficiency and this suggests chemical adsorption mechanism [35]. 
The relationship between the corrosion rate $(C R)$ of mild steel and aluminium in acidic medium and temperature (T) is often expressed by the Arrhenius equation:

$$
C R=A \exp \left(-E_{a} / R T\right)
$$

$E_{a}$ is the activation energy, $A$ the preexponential factor and $R$ the universal gas constant. The variation of logarithm of corrosion rate with reciprocal of absolute temperature for mild steel and aluminium is shown in Fig. (12). The calculated values of $E_{a}$ are presented in Table 4. Addition of HEC is seen to decrease the apparent activation energy for the corrosion reaction in $0.5 \mathrm{M} \mathrm{H}_{2} \mathrm{SO}_{4}$, implying that the compound is more effective at high temperatures.

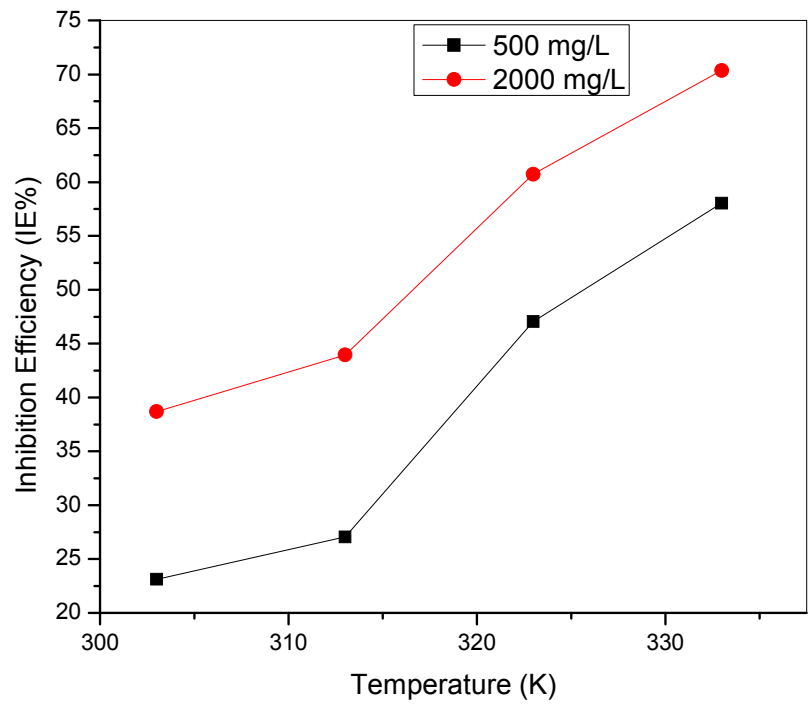

Fig. (10). Inhibition efficiency against temperature for mild steel corrosion in $0.5 \mathrm{M} \mathrm{H}_{2} \mathrm{SO}_{4}$.

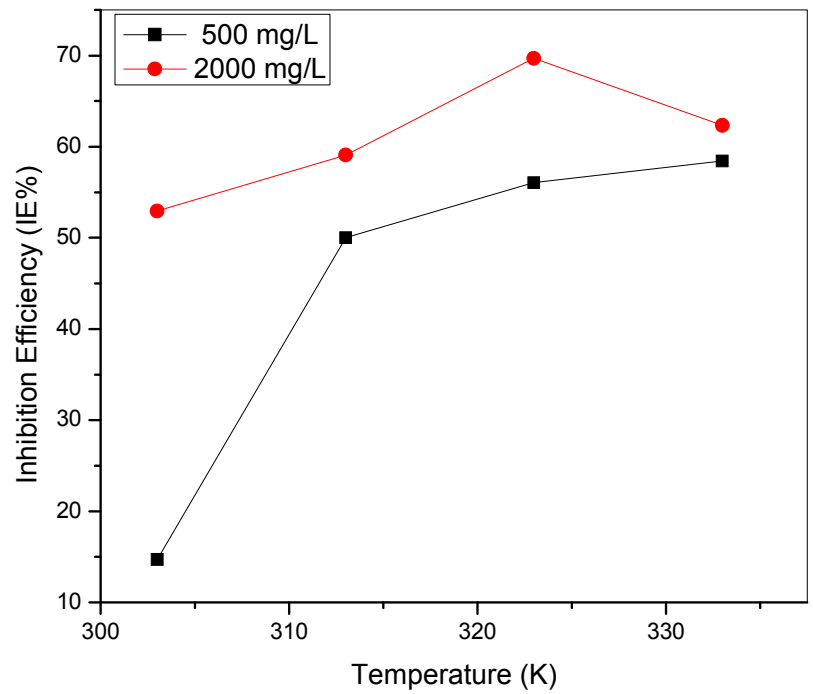

Fig. (11). Inhibition efficiency against temperature for aluminium corrosion in $0.5 \mathrm{M} \mathrm{H}_{2} \mathrm{SO}_{4}$.

According to Dehri and Ozcan [34] the relationship between the temperature dependence of inhibition efficiency of HPMC inhibitor and the activation energy found in its presence was given as follows:

i). For the inhibitors whose IE\% decreases with increasing temperature, the value of activation energy found is greater than that in the uninhibited solution

ii). For the inhibitors whose IE\% does not change with temperature variation, activation energy does not change with the presence or absence of inhibitors

iii). For the inhibitors whose IE\% increases with increasing temperature, the value of activation energy is less than that in the uninhibited solution.

However, Oguzie, et. al. [35] reported that decrease in $\mathrm{IE} \%$ with increasing temperature is an indication of physical adsorption whereas increase in inhibition efficiency with rise in temperature suggests chemical adsorption mechanism.
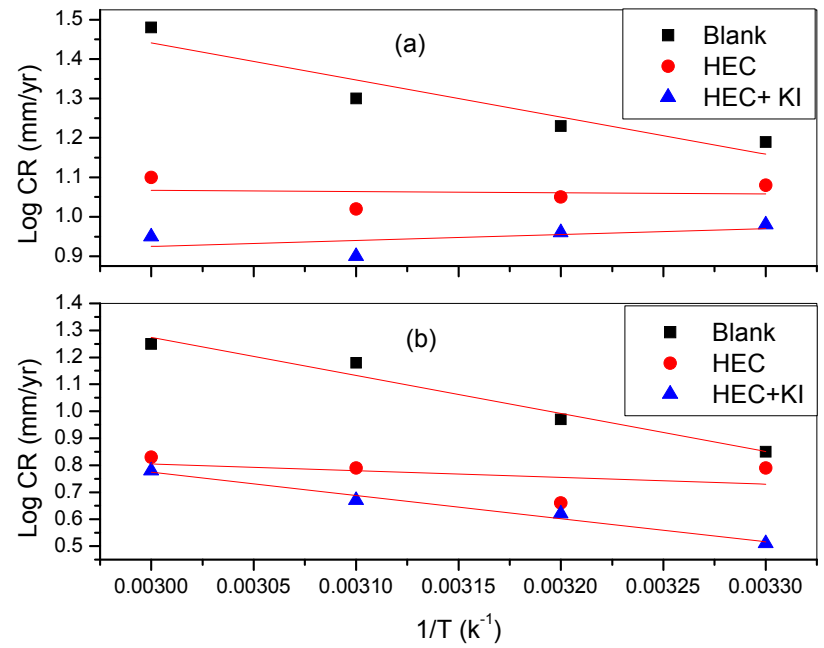

Fig. (12). Arrhenius plots for (a) mild steel and (b) aluminium corrosion in $0.5 \mathrm{M} \mathrm{H}_{2} \mathrm{SO}_{4}$ in the presence of HEC and HEC+KI.

Table 4. Calculated Values of Activation Energy for (a) Mild Steel and (b) Aluminium Corrosion in $0.5 \mathrm{M} \mathrm{H}_{2} \mathrm{SO}_{4}$

\begin{tabular}{|c|c|}
\hline System (mg/L) & $E_{a}(\mathrm{~kJ} / \mathrm{mol})$ \\
\hline (a) Blank & 7.82 \\
\hline HEC & 0.25 \\
\hline HEC + KI & -1.25 \\
\hline (b)Blank & 11.72 \\
\hline HEC & 2.08 \\
\hline HEC+ KI & 7.15 \\
\hline
\end{tabular}

\subsection{Computational Details}

\subsubsection{Quantum Chemical Computation}

The motivation for the computational studies is to gain insight into the theoretical framework in which to recognize the relationships between electronic structure and corrosion inhibition performance. Density functional theory (DFT) computations were thus performed to model the electronic structure of HEC. The effectiveness of inhibitors with different chemical structures have been correlated with the 


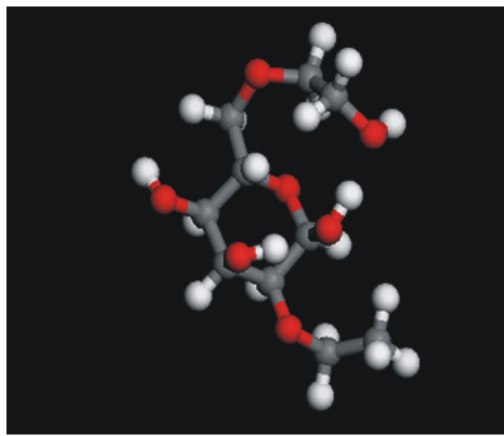

(A) Optimized Structure

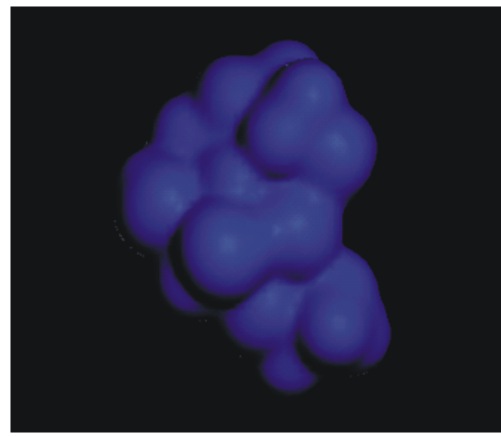

(D) Total electron density

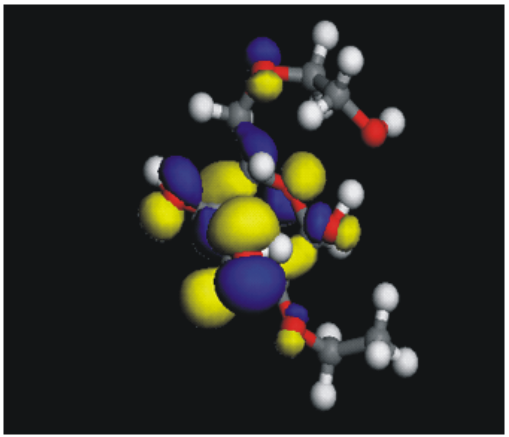

(B) HOMO Orbital

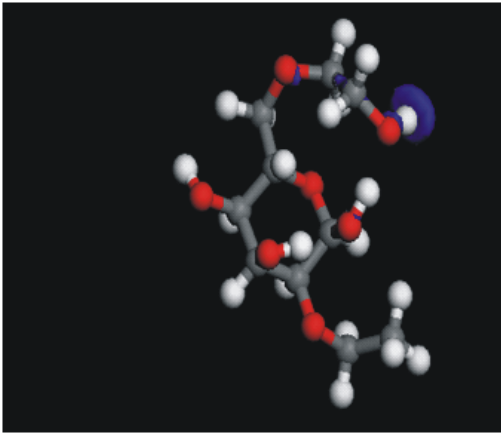

(E) Fukui function for Nuclephilic attack

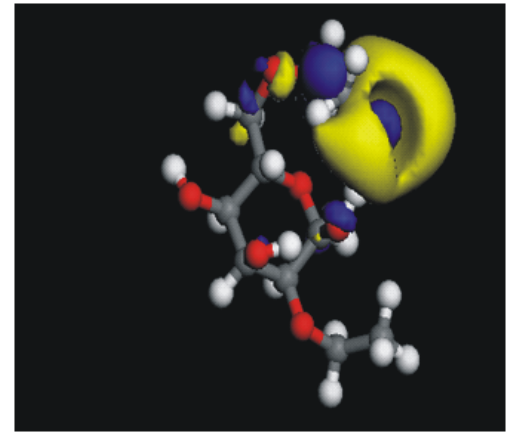

(C) LUMO Orbital

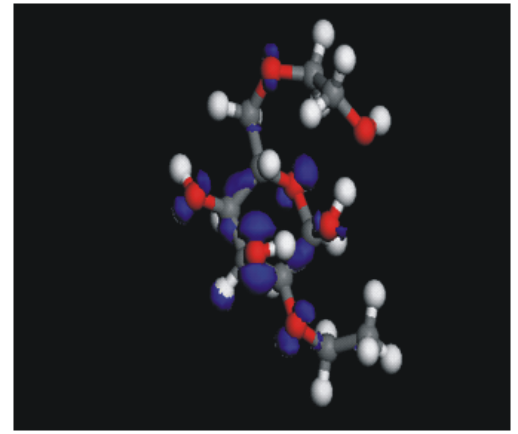

(F) Fukui function for electrophilic attack

Fig. (13). Electronic distribution of hydroxyethyl cellulose (HEC) [C, grey; H, white; O, red].

quantum chemical parameters such as energy of HOMO (Highest Occupied Molecular Orbital), LUMO (Lowest Unoccupied Molecular Orbital) and the energy gap between the LUMO and HOMO ( $\left.\Delta \mathrm{E}=\mathrm{E}_{\mathrm{LUMO}}-\mathrm{E}_{\mathrm{HOMO}}\right)$ [36]. High HOMO values are associated with the capacity of a molecule to donate an electron to an appropriated acceptor with empty molecular orbital, which facilitate the adsorption process and therefore indicates better performance of the corrosion inhibitor. $\mathrm{E}_{\mathrm{LUMO}}$ corresponds to a tendency for electron acceptance usually from the metal surface atoms. Based on this, the calculated difference $\left(\mathrm{E}_{\mathrm{LUMO}}-\mathrm{E}_{\mathrm{HOMO}}=5.45 \mathrm{eV}\right)$ demonstrates inherent electron donating ability and measures the interaction of the inhibitor molecule with the metal surface. The electronic structure of HEC, the molecular orbitals and Fukui indices have been modeled in order to establish the active sites as well as local reactivity of the molecule.

The geometry optimized structure of HEC molecule, HOMO and LUMO orbitals, Fukui function and the total electron density are depicted in Fig. (13). The HOMO and LUMO energies for HEC were $-5.589 \mathrm{eV}$ and $-0.139 \mathrm{eV}$ respectively. The high value of $\mathrm{E}_{\mathrm{HOMO}}$ suggests that $\mathrm{HEC}$ has high capacity to be readily adsorbed on the metal surface. The $\Delta \mathrm{E}$ value $(5.45 \mathrm{eV})$ again indicates high reactivity of the molecule with respect to adsorption on the mild steel and aluminum surfaces. Local reactivity was analyzed by means of the Fukui indices (FI) to assess reactive regions in terms of nucleophilic and electrophilic behaviour. Thus, the site for nucleophilic attack will be the place where the value of $\mathrm{F}^{+}$is maximum which corresponds to the HOMO locations. In turn, the site for electrophilic attack is controlled by the value of $\mathrm{F}^{-}$which coincides with the LUMO locations.

\subsubsection{Molecular Dynamics (MD) Simulation}

Molecular dynamics (MD) simulation was also performed to understudy the interaction between the HEC inhibitor molecule and the corroding metal surface to probe the inhibitor adsorption process at the molecular level. Simulations were performed using Forcite quench molecular dynamics in the MS modeling 4.0 software, to sample many

different low energy minima and to determine the global energy minimum. Fig. (14) shows different conformations of the lowest energy adsorption model for a single HEC molecule on Fe ( $\left(\begin{array}{lll}1 & 1 & 0\end{array}\right)$ and $\mathrm{Al}\left(\begin{array}{lll}1 & 1 & 0\end{array}\right)$ surfaces from the simulation.

The corresponding adsorption energies were computed as follows:

$$
E_{\text {ads }}=E_{\text {total }}-\left(E_{\text {inh }}+E_{\text {metal }}\right)
$$

The obtained values were -119.5 and $-52.80 \mathrm{eV}$ for HEC adsorption on mild steel and aluminium respectively. The adsorption values of HEC on mild steel and aluminum 

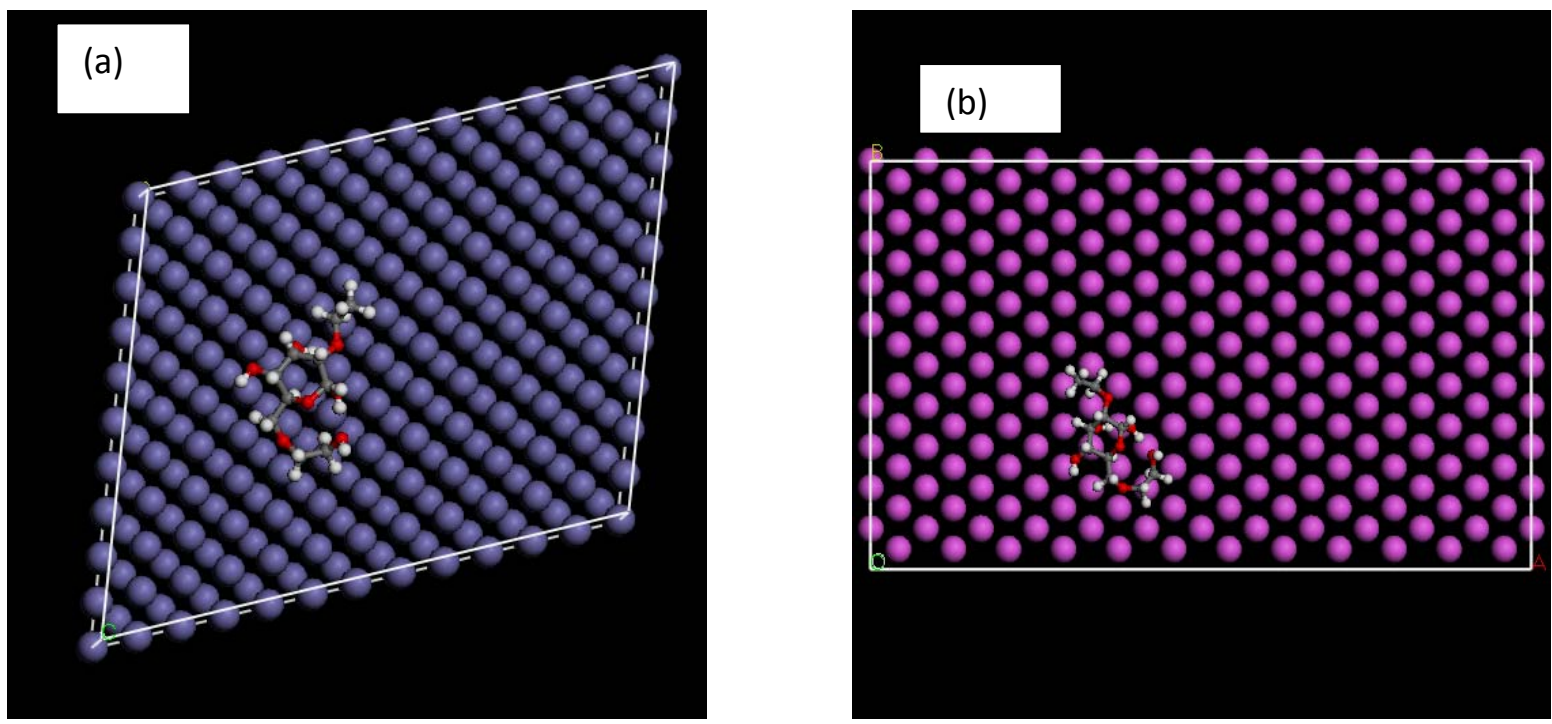

Fig. (14). Mode of adsorption of HEC on (a) Fe (110) surface and (b) Al (110) surface.

suggest that HEC should normally be strongly adsorbed more readily on a corroding mild steel surface than on the aluminum surface. The lower adsorption rate of aluminium could be related to the passive oxide film formed on the aluminium surface in the test solution, which interacts more favourably with active sites on the aluminium surface and renders them unavailable for inhibitor adsorption. Therefore, any observed corrosion inhibiting effect will result from simple geometrical blocking of the cathodic sites on the aluminium surface. Corroborating the experimental results, the theoretical studies undertaken show that the adsorption energy of HEC on mild steel surface is more exothermic, indicating higher inhibiting reaction than on aluminium surface.

\section{CONCLUSIONS}

Hydroxyethyl cellulose was examined as corrosion inhibitor for mild steel and aluminium in $0.5 \mathrm{M} \mathrm{H}_{2} \mathrm{SO}_{4}$ solution. The compound was found to inhibit the corrosion process by adsorption on the metal/solution interface. Inhibition efficacy increased with rise in temperature while the apparent activation energy decreased in the presence of inhibited systems, which suggest chemical adsorption mechanism. Inhibition efficiency was also found to depend on the concentration of inhibitor. HEC was more effective in mild steel than in aluminium. Polarization studies show that HEC is a mixed-type inhibitor for mild steel corrosion while it only inhibited cathodic reaction of aluminium. Decrease in the values of double layer capacitance with corresponding increase in interfacial layer is a clear confirmation of adsorption of HEC on the metal surfaces. Adsorption of HEC on the metal surface followed Langmuir and Freundlich isotherms for aluminium and mild steel respectively. Quantum chemical calculation was performed to describe adsorption of HEC at a molecular level. The quantum chemical descriptors show that HEC should be readily adsorbed on the corroding mild steel and aluminium surfaces.

\section{CONFLICT OF INTEREST}

The authors confirm that this article content has no conflict of interest.

\section{ACKNOWLEDGEMENTS}

The assistance from Electrochemistry and Material Science Research Unit (EMRU), Department of Chemistry, Federal University of Technology, Owerri, Nigeria is gratefully acknowledged.

\section{REFERENCES}

[1] Abiola OK, James AO. The effects of Aloe vera extract on corrosion and kinetics of corrosion process of zinc in $\mathrm{HCl}$ solution. Corros Sci 2010; 52: 661-4.

[2] Oguzie EE, Onuoha G, Onuchukwu, AI. Inhibitory mechanism of mild steel corrosion in $2 \mathrm{M}$ sulphuric acid solution by methylene blue dye. Mater Chem Phys 2005; 89: 305-11.

[3] Abdallah M. Ethoxylated fatty alcohols as corrosion inhibitors for dissolution of zinc in hydrochloric acid. Corros Sci 2003; 45: 270516.

[4] Abiola OK, Otaigbe JOE. The effects of Phyllanthus amarus extract on corrosion and kinetics of corrosion processes of aluminium in alkaline solution. Corros Sci 2009; 51: 2790-3.

[5] Abiola OK, Otaigbe JOE. Adsorption Behaviour of 1-phenyl-3methylpyrazol-5-one on mild steel from $\mathrm{HCl}$ solution. Int $\mathrm{J}$ Electrochem Sci 2008; 3: 191-8.

[6] Zhang Z, Chen S, Li Y, Li S, Wang L. A study of the inhibition of iron corrosion by imidazoline and its derivatives self-assembled films. Corros Sci 2009; 51: 291-300.

[7] Arshadi MR, Lashgari M, Parsafar GA. Cluster approach to corrosion inhibition problems: interaction studies. Mater Chem Phys 2004; 86: 311-4.

[8] Oguzie EE, Unaegbu C, Ogukwe CN, Okolue BN, Onuchukwu AI. Inhibition of mild steel corrosion in sulphuric acid using indigo dye and synergistic halide additives. Mater Chem Phys 2004; 84: 363

[9] Stupnisek-Lisac E, Podbrscek S, Soric T. Non-toxic organic zinc corrosion inhibitors in hydrochloric acid. J Appl Electrochem 1994; 24: 779-84.

[10] Abiola OK, Otaigbe JOE, Kio OJ. Gossipium hirsutum L. extracts as green corrosion inhibitor for aluminium in $\mathrm{NaOH}$ solution. Corros Sci 2009; 51: 1879-81.

[11] Abdel-Gaber A, Khamis E, Abo-Eldahab H, Adeel S. Inhibition of aluminium corrosion in alkaline solutions using natural compound. Mater Chem Phys 2008; 109: 297-305. 
[12] James OA, Atela AO. The effects of Aloe vera extract on corrosion and kinetics of corrosion process of zinc in $\mathrm{HCl}$ solution. Int $\mathrm{J}$ Pure Appl Chem 2008; 3: 159- 63.

[13] Ahamad I, Prasad R, Quraishi MA. Inhibition of mild steel corrosion in acid solution by pheniramine drug: Experimental and theoretical study. Corros Sci 2010; 52: 3033-41.

[14] Obot IO, Obi-Egbedi NO. Inhibitive properties, thermodynamic and quantum chemical studies of alloxazine on mild steel corrosion in $\mathrm{H}_{2} \mathrm{SO}_{4}$. Corros Sci 2011; 53: 263-75.

[15] Ahamad I, Quraishi MA. Mebendazole: New and efficient corrosion inhibitor for mild steel in acid medium. Corros Sci 2010; 52: 651-6.

[16] Musa AY, Kadhum AH, Mohamad AB, Takriff MS, Daud AR, Kamarudin SK. Inhibition of mild steel corrosion by 4-amino-5phenyl-4H-1,2,4-triazole-3-thiol. Corros Sci 2010; 52: 526-33.

[17] Singh AK, Quraishi MA. Effect of Cefazolin on the corrosion of mild steel in $\mathrm{HCl}$ solution. Corros Sci 2010; 52: 152-60.

[18] Hussain MH, Kassim MJ. The corrosion inhibition and adsorption behaviour of Uncaria gambir extract on mild steel in $1 \mathrm{M} \mathrm{HCl}$. Mater Chem Phys 2010; doi: 10.1016/j.matchemphys.2010.10.032.

[19] Lebrini M, Robert F, Vezin H, Roos C. Electrochemical and quantum chemical studies of some indole derivatives as corrosion inhibitors for C38 steel in molar hydrochloric acid. Corros Sci 2010; 52: 3367-76.

[20] Outirite M, Lagrenee M, Lebrini M, et al. ac impedance, x-ray photoelectron spectroscopy and density functional theory studies of 3,5-bis (n-pyridyl)-1,2,4-oxadiazoles as efficient corrosion inhibitors for carbon steel surface in hydrochloric acid solution. Electrochim Acta 2010; 55: 1670-81.

[21] Jianguo J, Lin W, Otieno-Alego V, Schweinsberg DP. Polyvinyl pyrrolidone and Polyethylenimine as corrosion inhibitors for the corrosion of a low carbon steel in phosphoric acid. Corros Sci 1995; 37(6): 975-85.

[22] Dubey AK, Singh G. Corrosion Inhibition of mild steel by using cetyl pyridinium bromide (CPB). Port Electrochim Acta 2007; 25: $249-62$.

[23] Rajendran S, Sridevi SP, Anthony N, Amalraj JA, Sundearavadivelu M. Corrosion behaviour of carbon steel in Polyvinyl alcohol. Anti-Corrosion Methods Mater 2005; 52(2): 102-7.
[24] Kumpawat V, Garg U, Tak RK. Corrosion Inhibition of aluminium in acid media by naturally occurring plant Artocarpus heterophyllus and Acacia Senegal. J Ind Council Chem 2009; 26(1): 82-4.

[25] Bockris JOM, Drazic D. The electrode kinetics of the deposition and dissolution of iron: Effect of alloying impurities. Electrochim Acta 1962; 7: 293

[26] Oguzie EE. Influence of halide ions on the inhibitive effect of congo red dye on the corrosion of mild steel in sulphuric acid solution. Mater Chem Phys 2004; 87: 212-7.

[27] Ashassi-Sorkhabi H, Nabavi-Amri SA. Corrosion inhibition of carbon steel in petroleum /water mixtures by n-containing compounds. Acta Chim Slov 2000; 47: 507-17.

[28] Foley RT, Nguyen TH. The chemical nature of aluminium corrosion V. Energy transfer in aluminium dissolution. J Electrochem Soc 1982; 129(3): 464-7.

[29] Foley RT. Localized corrosion of aluminium alloys - a review. Corrosion 1986; 42: 277.

[30] Wong KP, Alkire RC. Local chemistry and growth of single corrosion pits in aluminium. J Electrochem Soc 1990; 137(10): 3010-5.

[31] Hosseini M, Mertens SF, Arshadi MR. Synergism and antagonism in mild steel corrosion inhibition by Sodium dodecylbenzenesulphonate and hexamethylenetetramine. Corros Sci 2003; 45: 1473.

[32] Obot IB, Obi-Egbedi NO. Adsorption properties and inhibition of mild steel corrosion in sulphuric acid solution by ketoconazole: Experimental and theoretical investigation. Corros Sci 2010; 52: 198-204.

[33] Lyberatos G, Kobotiatis L. Inhibition of aluminium 7075 alloy corrosion by the concerted action of nitrate and oxalate salts. Corrosion 1991; 47: 820 .

[34] Dehri I, Ozcan M. The effect of temperature on the corrosion of mild steel in acidic media in the presence of some sulphurcontaining organic compounds. Mater Chem Phys 2006; 98: 31623 .

[35] Oguzie EE, Njoku VO, Enenebeaku CK, Akalezi CO, Obi C. Effect of hexamethylpararosaniline chloride (crystal violet) on mild steel corrosion in acidic media. Corros Sci 2008; 50: 3480-86.

[36] Oguzie EE, Wang SG, Li Y, Wang FH. Influence of iron microstructure on corrosion inhibitor performance in acidic media. J Phys Chem C 2009; 113: 8420-9.

(C) Arukalam et al.; Licensee Bentham Open.

This is an open access article licensed under the terms of the Creative Commons Attribution Non-Commercial License (http://creativecommons.org/licenses/ by-nc/3.0/) which permits unrestricted, non-commercial use, distribution and reproduction in any medium, provided the work is properly cited. 